\title{
IL FASCINO DEL FIABESCO: LA FIABA TRA PASSATO E PRESENTE
}

\author{
Elis Deghenghi Olujić \\ Università degli Studi Juraj Dobrila di Pola (Croazia) \\ elis.olujic@pu.t-com.hr
}

\begin{abstract}
Riassunto
Il presente saggio s'incentra sulla fiaba, genere presente in tutte le culture, alla quale è stato affidato, sin dai primordi, il compito di parlare a livello simbolico dell'essenza stessa dell'esperienza umana. Con antica saggezza e trasparenza di significati la fiaba risponde alle domande centrali della vita: la nascita, l'amore, la morte. Le sue caratteristiche formali (l'assenza di denominazioni, l'atemporalità, l'astrattezza delle figure e la mancata definizione dello spazio, l'uso dei simboli, l'universalità) le permettono di trascendere la storicità dell'esistenza per radicarsi in quella dimensione che appartiene alla vita immaginativa e inconscia del fruitore, basata appunto sui simboli.
\end{abstract}

Parole chiave: fiaba, fiabesco, tradizione popolare, fantasia, creatività 


\section{Introduzione}

Il presente saggio trae spunto dal giudizio espresso da Gianni Rodari (1920-1980) a proposito della fiaba. L'autore della Grammatica della fantasia, nella sua vastissima produzione spaziante dalla fiaba moderna alla poesia alla saggistica, ha dedicato ampio spazio all'analisi della fiaba classica e l'ha definita "materia prima" del narrare e dello scrivere ${ }^{[1]}$. Presente in tutte le culture fin dai primordi, la fiaba ha mostrato nei secoli una straordinaria disponibilità alla metamorfosi. Questa estrema malleabilità le ha consentito di resistere nel tempo, passando di mano in mano, adeguandosi alle diverse intenzioni degli autori, adattandosi alle mode, agli stili e ai diversi linguaggi (si pensi al cinema e al teatro), per arrivare fino alla nostra epoca a mostrarci, ancora intatta, la sua valenza educativa e a confermarci una verità ampiamente assodata e riconosciuta: la fiaba è la fonte primaria del narrabile ${ }^{[2]}$. Nella sua elementare semplicità essa rimane pertanto una delle più straordinarie espressioni della creatività umana.

[1] Rodari si è interessato ai problemi della «fantastica» fin dagli anni giovanili, come ricorda nella Grammatica della fantasia. Leggendo i Frammenti di Novalis, sul finire degli anni Quaranta dello scorso secolo, ha scoperto il numero 1095, che afferma: "Se avessimo anche una Fantastica, come una Logica, sarebbe scoperta l'arte di inventare". È in quel tempo che egli dice di aver intitolato "pomposamente un modesto scartafaccio" Quaderno di Fantastica, annotando in esso non le storie raccontate ai suoi allievi (all'epoca insegnava l'italiano ai bambini di una famiglia di ebrei tedeschi, che credevano di aver trovato in Italia il rifugio alle persecuzioni razziali), ma il modo in cui nascevano, i trucchi che scopriva o credeva di scoprire per mettere in movimento le parole e le immagini. Questo Quaderno fu dimenticato e accantonato fino al 1948, quando Rodari inizia a scrivere per bambini. Le radici della «fantastica» rodariana stanno anche in un'altra esperienza, che Rodari realizza nel 1962, quando pubblica sul quotidiano romano «Paese sera» un Manuale per inventare favole. Il titolo mostra che, anche Rodari, come molti, indica impropriamente la «fiaba»col termine «favola», che è un genere narrativo diverso, che consiste in un componimento letterario di tono e intento satirico, sottilmente metaforico, in cui animali, piante o elementi naturali, interloquiscono e agiscono come uomini, enunciando un precetto morale o di saggezza pratica. Anche se i due generi hanno in comune il carattere narrativo, l'origine popolare e l'etimologia (dal latino «fabula»), essi differiscono nettamente sotto il profilo della struttura, del contenuto e della finalità. Appare pertanto del tutto ingiustificata, da parte di persone di cultura e in manuali di carattere non divulgativo bensì specialistico, la confusione spesso presente nell'uso dei termini con cui i due generi sono designati.

[2] Italo Calvino, l'autore che ci ha regalato le Fiabe italiane (1956), ha definito la fiaba una sterminata "campionatura della molteplicità potenziale del narrabile", una macchina per moltiplicare le narrazioni, un universo formato di pochi elementi ma tutti in continua trasformazione. Sarà proprio lavorando sulla fiaba che l'arte della narrativa assumerà per Calvino i caratteri e le prerogative di un'arte combinatoria, messa a punto nel Castello dei destini incrociati (1973). Come osserva Mario Lavagetto nell'Introduzione dell'opera Sulla fiaba da lui curata, che raccoglie in volume gli scritti di Calvino sul genere fiabesco, lo scrittore, nel quale il gusto per il meraviglioso era già evidente nel Sentiero dei nidi di ragno (1947) e nei primi racconti del 1946 e del 1947, ha fatto sue le qualità degli anonimi novellatori popolari: la fantasia, la precisione, la competenza narrativa, il perfetto controllo dei meccanismi, la sapienza descrittiva e nomenclatoria. Agli occhi di Calvino, come osserva ancora Lavagetto nella citata Introduzione, la fiaba si configura "come una grande enciclopedia del narrabile, di funzioni originarie che - più o meno trasformate - si possono ritrovare alle spalle di Chisciotte, di Amleto, di Robinson, di Fabrizio del Dongo" (pp. X e XI). In breve, secondo Calvino, la fiaba è un bene collettivo che ha già in sé tutte le caratteristiche della grande letteratura di tutti i tempi. Sempre secondo Lavagetto, tra la fiaba e il romanzo per Calvino non esiste "nessuna cesura netta, né frontiera invalicabile". Per l'autore de I nostri antenati (1960), conclude Lavagetto, la fiaba "è come una magica noce o nocciola o mandorla nel cui interno un potenziale scrittore di apocrifi può trovare non gioielli e ricchezza, ma l'intero universo della narrativa e le sue costellazioni" (p. XI). 
Alla fiaba, fin dalle sue origini, che si perdono nella notte dei tempi, è stato affidato il compito di parlare a livello simbolico dell'essenza stessa dell'esperienza umana, di rispondere con saggezza e trasparenza di significati alle questioni centrali della vita quali la nascita, l'amore, la morte, senza la pretesa di ammaestrare, imponendo come unica regola il ritmo del racconto e la sua intima coesione. Le fiabe, per Italo Calvino, "prese tutte insieme, nella loro ripetuta e sempre varia casistica di vicende umane", rappresentano "una spiegazione generale della vita, nata in tempi remoti e serbata nel lento ruminio delle coscienze contadine fino a noi; sono il catalogo dei destini che possono darsi a un uomo e a una donna, soprattutto per la parte di vita che appunto è il farsi d'un destino: la giovinezza, dalla nascita che sovente porta in sé un auspicio o una condanna, al distacco dalla casa, alle prove per diventare adulto e poi maturo, per confermarsi come essere umano" ${ }^{[3]}$. Le fiabe sono effettivamente un grande serbatoio di messaggi, un vastissimo patrimonio delle fantasie, delle speranze, delle credenze e delle paure degli uomini, che albergano ancora nella nostra modernità. I messaggi impliciti delle fiabe ripercorrono le angosce più complesse del genere umano quali la paura della morte, dell'abbandono, della fame, e propongono delle vie per comprenderle e superarle: quando ciò non è possibile con le sole forze umane, interviene la magia, che rappresenta la capacità di non rassegnarsi bensì di comprendere il problema e superarlo interiormente.

Le caratteristiche formali di quest'antico genere quali l'assenza di denominazioni, l'atemporalità, l'astrattezza delle figure, l'uso dei simboli, l'universalità, le permettono di trascendere la storicità dell'esistenza per collocarsi in quella dimensione che appartiene all'inconscio e alla nostra vita immaginativa, basata sui simboli. La fiaba non racconta, infatti, soltanto una storia, bensì raccoglie in sé una serie di dati conoscitivi e interpretativi presi dalla realtà culturale che, dopo molte trasformazioni e un lungo processo di sviluppo e di adeguamento durante l'arco dei secoli, hanno assunto l'aspetto del simbolo. Ed è proprio su questo piano simbolico che la fiaba, scissa ormai dalla sensibilità della cultura di massa, continua a sopravvivere nella moderna civiltà tecnologica come uno dei generi che l'infanzia, in primis, ha fatto proprio.

[3] Calvino 1988a: 19. 
In origine e fino al XVII secolo le fiabe venivano raccontate sia agli adulti sia ai bambini. Per secoli la fiaba è stata un genere d'intrattenimento che coinvolgeva l'intera comunità: è evidente quindi che essa ha una matrice popolare. Nella cultura occidentale moderna, la fiaba è stata spinta alla periferia del sistema letterario e "abbandonata" alla letteratura per l'infanzia. A detta di Rodari, la fiaba è stata ceduta all'infanzia per "caduta", ovvero in seguito alla perdita di significato nel mondo degli adulti. I bambini sono diventati i fruitori ideali della fiaba in quanto essa è particolarmente adatta alla loro mentalità, sia per i temi trattati sia per le sue caratteristiche formali, ed esige che il destinatario accetti la narrazione senza provare dissonanza cognitiva o assumere un atteggiamento critico. Per il lettore adulto, invece, che con il passar del tempo è diventato più sofisticato e smaliziato e si è munito di un bagaglio di conoscenza notevole, è risultato più difficile calarsi nel mondo fiabesco eliminando ogni traccia di dubbio per trarre piacere unicamente dal nucleo narrativo elementare e prevedibile della fiaba. Tuttavia, proprio perché la fiaba è "un genere narrativo fatto apposta per essere usato, ossia piegato ai fini specifici del fruitore ed adattato alle situazioni particolari in cui si trova di volta in volta ad esistere" ${ }^{[4]}$, essa può favorire e sopportare nuove rielaborazioni e riscritture. Non stupisce pertanto che molti autori riscrivano le fiabe classiche mirando a evidenziare contenuti anche marginali, per proporli in chiave insolita e personale, o ne scrivano di nuove, adeguandole agli interessi del bambino contemporaneo (oltre a Rodari, Marcello Argilli, Pinin Carpi, Beatrice Solinas Donghi, Roberto Piumini, Bianca Pitzorno). Aggiungendo alla prospettiva personale i contributi provenienti da saperi molteplici e da determinati campi della cultura, concedendosi un notevole margine di libertà interpretativa, l'adulto può oggi affrontare la lettura della fiaba seguendo diversi percorsi: antropologico, psicoanalitico, etnologico, mitologico, storico-ideologico. La fiaba classica diventa così, oltre che fonte cui attingere per trovare spunti narrativi da rielaborare in chiave moderna, oggetto di studio di molte discipline: la filologia, l'etnologia, la sociologia, l'antropologia, la semiologia, la psicanalisi, la critica letteraria, la storia delle religioni.

[4] Pisanty 1993: 5-6. 


\section{La nascita del genere fiabesco: la raccolta Kinder und Hausmärchen dei fratelli Grimm}

Le fiabe nascono dalla tradizione orale. Di questa tradizione conservano alcune caratteristiche strutturali fondamentali, tanto che ancora oggi vengono raccontate e ascoltate piuttosto che lette. Le fiabe sono prima di tutto "racconti", e come tali costituiscono il primo approccio del bambino alla letteratura propriamente detta ${ }^{[5]}$. Dal momento in cui un autore trascrive una fiaba dalla tradizione orale, essa perde la sua forma pura e incontaminata e si trasforma da "forma semplice" in "forma artistica”. La fiaba cessa, da quel momento, d'essere lo specchio dei processi psichici della collettività e assume l'impronta dell'autore ${ }^{[6]}$. Si fa per questo distinzione tra la fiaba d'estrazione popolare e la fiaba colta, nella quale il fantastico e il meraviglioso si dilatano, dando spazio a creature immaginarie lontane dalla tradizione popolare che è più realistica, come le fate, e dando vita a un universo di metamorfosi assai intense, a itinerari esistenziali in cui l'orrore si allaccia al lieto fine e al risarcimento, alla realizzazione del sogno.

La fiaba letteraria moderna, come la conosciamo oggi, nasce con i fratelli Grimm. Jacob (1785-1863) e Wilhelm (1786-1859) vissero e lavorarono come studiosi di letterature antiche, filologi e bibliotecari nella prima metà dell'Ottocento nella Germania allora divisa in numerosi stati. Furono legati da rapporti d'amicizia con i rappresentanti della cosiddetta seconda generazione romantica e con essi contribuirono a creare un particolarissimo concetto di cultura popolare, di cui le fiabe sono una delle voci più caratteristiche. Nella loro operazione di raccolta del patrimonio fiabistico tedesco, inserito nella collezione Kinder und Hausmärchen, attinsero sia dalla tradizione orale popolare tedesca sia dalla tradizione scritta letteraria già esistente. Pubblicata in due volumi (il primo uscì nel 1812 e il secondo nel 1815) l'opera, tradotta con il titolo Fiabe per i bambini

[5] Questo concetto è stato espresso, oltre che da Michel Butor (La Balance des fées) anche da Beatrice Solinas Donghi e da Furio Iesi in Sul mito e sulla fiaba, in AA. VV., Tutto è fiaba, Emme, Milano, 1980, p. 46.

[6] Riferendosi ad André Jolles, che tra le "forme semplici", oltre alla fiaba, annovera ancora la leggenda, sacra e profana, il mito, la sentenza, l'enigma, il caso, il memorabile e lo scherzo, Valentina Pisanty a p. 30 di Leggere la fiaba, nell'analizzare il genere fiabesco, fa distinzione tra "forma semplice" e "forma artistica". La "forma semplice" corrisponde a una "autocreazione spontanea" che nasce dalla tradizione orale, la "forma artistica" è invece una "elaborazione", una "attualizzazione", che richiede "l'intervento creativo del singolo". Per esempio, sono considerate fiabe d'arte o d'autore, dunque "artistiche", le creazioni di Andersen, di Hoffmann, di Hauff, di Perrault. 
e per la casa, ebbe ben sette edizioni mentre i Grimm erano in vita, e subì vari ampliamenti, dovuti soprattutto a Wilhelm ${ }^{[7]}$. Questa raccolta, forse la più famosa al mondo, è molto più che una semplice raccolta di fiabe e la sua nascita, oltre a rappresentare un momento fondamentale nella storia della cultura, segna l'inizio stesso del genere letterario che correntemente chiamiamo "fiaba". I Kinder und Hausmärchen non sono il frutto di un lavoro svolto in una determinata direzione, bensì uno dei risultati estrapolati dell'attività complessiva dei due fratelli. Di certo, essi pensavano ai bambini come alla parte fruitrice ${ }^{[8]}$. Lo prova già la dedica alla prima edizione: "Alla signora Elizabeth von Arnim per il piccolo Johannes Freimund". Il piccolo era il primogenito, allora nato da poco, di Achim von Arnim, poeta tardoromantico tedesco autore, con Clemens Brentano, fratello di Elizabeth, della raccolta di canzoni popolari intitolata Il corno magico del fanciullo che, con qualche ritocco e abbellimento, doveva essere un testo educativo.

Leggendo la prefazione della prima edizione si desume che in realtà gli autori non avevano in mente solo un uso esclusivamente infantile e domestico delle loro fiabe. Ciò che in primo luogo premeva era portare alla luce questi fiori della fantasia poetica popolare fino a quel momento trascurati, e reintegrarli nel patrimonio nazionale sulla scia dell'esaltazione romantica delle radici profonde dello spirito nazionale, di cui il popolo sarebbe custode nelle sue tradizioni. Durante tutto il Settecento illuminista e razionalista, la fiaba era stata un genere disprezzato e "buono solo per le balie", accusato di riempire la testa con l'eccesso di fantasia. Con il primo Romanticismo, a cavallo tra Settecento e Ottocento, si assiste

[7] L'ultima edizione, quella del 1857, conta duecentouno fiabe, alle quali si aggiungono dieci cosiddette leggende per fanciulli. Il titolo della raccolta, difficilmente traducibile, merita alcune parole di approfondimento. Mentre il concetto di "fiabe per i bambini" emerge nel contesto del Romanticismo tedesco già prima dei fratelli Grimm, Hausmäechen (composto da Haus, casa, e Märchen, fiabe) parrebbe invece una loro creazione, uno di quei composti di cui la lingua tedesca è così ricca e il cui potere d'associazione va spesso molto al di là del loro preciso valore semantico. Il concetto di casa va interpretato come "famiglia allargata". La famiglia, intesa quale nucleo primario della società, nel processo di industrializzazione dell’Europa centrale, già all'inizio del XIX secolo, apparteneva sempre più al passato e diveniva per questo oggetto di trasfigurazione romantica. Va rilevato che in lingua italiana il titolo della raccolta grimmiana viene tradotto anche Fiabe per i bambini e il focolare, dove il termine "focolare" accentua ancor più l'idea della casa, della famiglia, e sottolinea che le fiabe sono quei racconti che si narravano un tempo seduti intorno al focolare.

[8] Appena lo lesse, Goethe lo definì un libro fatto apposta per rendere felici i bambini, e la sua fortuna tra le letture infantili nelle famiglie tedesche fu subito immensa. All'epoca non si parlava ancora della letteratura per l'infanzia secondo le caratteristiche che si elaborarono in seguito, soprattutto dopo la seconda metà dell'Ottocento. Appena una produzione di libri per l'infanzia prese corpo, i volumi dei Grimm diventarono fonte d'innumerevoli traduzioni, e le loro fiabe divennero patrimonio dell'infanzia in tutta Europa. Nella presentazione dell'edizione del 1837, nella nota a piè di pagina, i Grimm nominano gli autori delle numerose traduzioni della loro opera, prodotte fino a quel momento. Il lungo elenco ci dà la misura della fama di cui godeva il lavoro e della sua diffusione. 
invece a una vera e propria riscoperta della narrazione fiabesca. E se all'inizio la fiaba è soprattutto allegoria poetica, lieve invenzione in cui si trasfonde magicamente la potenza creatrice dell'ingegno umano e della parola poetica, per la seconda generazione romantica essa si trasforma in un prezioso documento della viva voce della poesia popolare. I Grimm consideravano le fiabe resti di antichi miti (espressero tale convinzione nella teoria mitica o mitologica sull'origine della fiaba), sopravvissuti nella memoria popolare e tramandati nella narrazione orale ${ }^{[9]}$. Il "popolo" è inteso dai fratelli e da tutto il secondo Romanticismo come un "organismo collettivo" (di "popolo novellatore" si parla nella teoria sull'origine della fiaba dei Grimm), definito non in base alla classe o al censo, ma in funzione di valori di appartenenza schietta alla società ed è quindi costituito da "uomini semplici", non corrotti, ancora vicini alle caratteristiche originarie e genuine del genere umano. Strettamente correlati a questa concezione del popolare e all'idea di una "poesia originaria" sono anche altri lavori dei due fratelli, benché certo meno noti delle Fiabe. Ricordiamo fra questi: Leggende tedesche (1816), Leggende tedesche degli eroi (1929, a cura di Wilhelm) e Mitologia tedesca (1835, a cura di Jacob).

Le condizioni che permettevano la trasmissione orale della cultura popolare, fiabe comprese, erano minacciate all'inizio dell'Ottocento da profondi mutamenti socio-economici che derivavano da un timido inizio di quel processo di industrializzazione che sarà più intenso in tutta Europa nella seconda metà dell'Ottocento, cambiamenti che interessavano anche la famiglia, cellula fondativa della società. Per ovviare a questo pericolo, come si annuncia nell'introduzione all'edizione del 1819 dei Kinder und Hausmärchen, curata da Wilhelm, era arrivato il momento di trascrivere le fiabe, di metterle per iscritto, poiché "coloro che devono conservarle" e trasmetterle erano "sempre più rari" ${ }^{[10]}$. L'opera nasce, almeno all'inizio

[9] Jacob, studioso e filologo, scrive all'amico Achim von Arnim, il 29 ottobre 1812: "Sono fermamente convinto che tutte le fiabe della nostra raccolta, con tutte le loro particolarità, venivano narrate già millenni fa. Con la sola differenza che poco alla volta è andato perduto qualcosa di bello. In questo senso tutte le fiabe si sono codificate come sono da lunghissimo tempo, mentre si spostano di qua e di là in infinite variazioni, cioè non codificandosi nelle singole variabili. Tali variazioni sono come i molteplici dialetti di una lingua e come quelli non devono subire forzature".

[10] Grimm 1999: 11. Le precedenti edizioni erano rivolte a un pubblico adulto e colto. A questa, del 1819, Wilhelm apportò ulteriori cambiamenti affinché i racconti si adeguassero alla sua immagine dell'infanzia ed esercitassero la funzione pedagogica che egli attribuiva ad essi. Come osserva Valentina Pisanty a p. 59 dell'opera Leggere la fiaba, "il pubblico borghese accolse questa iniziativa con tale entusiasmo che da quel momento in poi la raccolta grimmiana divenne una vera e propria istituzione nazionale (ma anche internazionale) attraverso la quale i bambini di molte generazioni assorbirono alcuni dei valori principali della cultura dominante. Le numerose raccolte di fiabe per bambini che hanno affollato il mercato editoriale 
e nelle intenzioni, come lavoro scientifico e filologico di raccolta e conservazione di un patrimonio popolare, senza finalità educative troppo esplicite. I Grimm intendevano stabilizzare e diffondere narrazioni che stavano per essere dimenticate. Nel farlo, dichiaravano di voler procedere con l'attenzione e la cura degli studiosi e non con la disinvoltura dei rielaboratori. Ma già il titolo, e ancor più i numerosi cambiamenti apportati nel corso delle varie edizioni, sono la testimonianza di un processo volto soprattutto a eliminare passi e dettagli "popolarmente" troppo crudi e inadatti al pubblico dei lettori, tra cui rientravano anche i bambini. Sempre nell'introduzione all'edizione del 1819, spiegando che la raccolta era scritta per i bambini e dunque anche con intento educativo, i Grimm precisano: "Questo è il motivo per cui con la nostra raccolta non abbiamo inteso soltanto rendere un servizio alla storia della poesia e alla mitografia; nostro intento, al tempo stesso, era far agire la poesia che in essa vive, far sì che essa allieti chi può allietare; che la raccolta, dunque, serva anche da libro educativo. [...] Noi cerchiamo la purezza nella verità di un racconto schietto, che nulla di ciò che è male nasconde con riserve. In questa nuova edizione abbiamo però accuratamente cancellato qualsiasi espressione non adatta all'infanzia" ${ }^{[1]}$.

Pur proponendosi di "rendere fedelmente con la scrittura la parola del popolo" ${ }^{[12]}$, l'intervento dei fratelli Grimm sul repertorio fiabistico non passa inosservato. Talvolta i due fratelli integravano le varianti della medesima fiaba in un'unica versione, introducendo delle aggiunte personali, ritoccando espressioni e immagini, dando unità di stile alle voci discordanti. Così spiegano il metodo adottato nella più ampia tra le introduzioni, quella del 1819: "Nel caso di narrazioni diverse che si integravano tra loro, se si potevano unificare senza dover tagliare parti contraddittorie, abbiamo dato una narrazione unica: se invece sussistevano differenze, nel qual caso ciascuna aveva di solito i suoi tratti specifici, abbiamo preferito la migliore e conservato le altre per le note" ${ }^{[13]}$. Con il loro intervento sul materiale fiabistico della tradizione popolare, essi conferirono ai racconti maggiore unità stilistica e una più salda coerenza, soprattutto per merito di Wilhelm, più ricco di sensibilità e fantasia, più

a partire dalla seconda metà dell'Ottocento hanno generalmente seguito le tracce della raccolta grimmiana".

[11] Grimm 1999: 12-13.

[12] Calvino 1988a: 84.

[13] Grimm 1999: 17-18. 
poeta rispetto a Jacob, filologo e scienziato, che stabilì invece i criteri con cui il lavoro andava svolto ${ }^{[14]}$.

Secondo quanto raccontano i due fratelli, la fonte principale che fornì loro la maggior parte delle fiabe è stata "frau Viehmännin", una contadina conosciuta in un villaggio nelle vicinanze di Kessel, che "aveva da poco passato la cinquantina ed era ancora gagliarda. Aveva qualcosa di solido, assennato e gradevole nei tratti, e i suoi grandi occhi guardavano limpidi e acuti" [15]. Questa donna è stata la loro "informatrice" più affidabile. Aveva una sorprendente memoria e raccontava "pensosa, sicura, con una straordinaria vivacità e provando gusto nel raccontare, una prima volta a braccio, poi, a richiesta, una seconda volta lentamente, in modo che prendendoci la mano si poteva trascrivere il racconto. Molte cose, in questo modo, sono state riportate letteralmente e risulteranno inconfondibili nella loro verità" ${ }^{[16]}$. Il passo qui riportato è indicativo del modo con cui i Grimm lavoravano. Pur concedendosi un margine di manovra, i due fratelli erano guidati dalla passione per la fedeltà scrupolosa alla fonte e alla versione originale, perché credevano che nei racconti della povera gente illetterata fossero nascoste una bellezza e una verità che bisognava riportare alla luce intatte. Quello che uscì dalla loro penna ha rappresentato e rappresenta ancora il tono fiabesco per eccellenza ed è, in questo senso, davvero "popolare", testimonianza della profonda sensibilità dei due curatori e della straordinaria forza vivificante della loro opera.

La raccolta grimmiana è servita da modello per intere generazioni di folcloristi o, con un termine più moderno, demologi. La sua pubblicazione stimolò in tanti paesi la raccolta sistematica delle fiabe nazionali e ben presto si rilevò che molti motivi narrativi, ritenuti

[14] Presentando i due fratelli nel saggio Le fiabe del focolare (in Sulla fiaba, a cura di Mario Lavagetto, p. 83), Calvino scrive come se a sua volta raccontasse una fiaba: "Gli autori di questo libro di fiabe sono due fratelli che, inseparabili fin da bambini, vissero e studiarono e scrissero insieme per tutta la loro vita. Il maggiore, Jacob (1785-1863) era il più ostinato e severo; il secondo, Wilhelm (1786-1859) il più gaio e il più poeta". In verità i due fratelli si completavano a vicenda. Jacob era il più vicino all'atteggiamento scientifico, era il ricercatore e il filologo rigoroso, che portò avanti con meticolosità l'arduo lavoro di raccolta del materiale fiabistico. Wilhelm era l'artista, che con il suo estro creativo "diede alla scrittura quella leggerezza e spontaneità che, quanto più ci appaiono naturali, tanto più sono il risultato d'un'arte", sottolinea Calvino. Lo scrittore conclude la biografia dei Grimm con lo stesso tono fiabesco con cui l'aveva iniziata. A pp. 8687 del saggio sopra citato scrive: "Nel 1825 Wilhelm si sposò; Jacob restò scapolo; ma non per questo i due fratelli si separarono: Jacob andò ad abitare con gli sposi. Come separarsi, del resto? Fin da ragazzi erano abituati a lavorare prima allo stesso banco, poi a due scrivanie nella stessa stanza. E i libri, soprattutto: tutti i loro libri erano proprietà comune di entrambi, e tutta la loro vita essendo nei libri, Jacob e Wilhelm erano più inseparabili di due fratelli siamesi".

[15] Grimm 1999: 15. Di questa donna, come ci informano gli stessi Grimm, esiste un ritratto eseguito da Ludwig Grimm, fratello di Jacob e Wilhelm, che si trova nella raccolta dei suoi disegni, pubblicata da Weigel, a Lipsia.

[16] Grimm 1999: 15. 
prodotto genuino della nazione tedesca, si ritrovavano in tutta l'Europa ed anche nel mondo islamico e indiano. Verso le fiabe sorse un interesse spiccatamente storico e scientifico, che spinse molti studiosi a tentare di spiegare quando e dove esse avevano avuto origine e perché vi fossero nelle fiabe motivi ricorrenti. Nacquero così molte teorie che cercavano di spiegare in vario modo la genesi della fiaba e di collocarla nello spazio e nel tempo, anche se a nostro avviso collocare la fiaba nel tempo e nello spazio è impossibile. Condividiamo il pensiero espresso da Italo Calvino, che ha lasciato importanti contributi sull'argomento. Nel saggio La tradizione popolare nelle fiabe, fin dalle prime righe, Calvino dichiara nettamente che "il racconto di meraviglie magiche, dal "c'era una volta" iniziale alle varie formule di chiusura, non ammette d'essere situato nel tempo e nello spazio" ${ }^{[17]}$.

Il lavoro avviato dai Grimm trovò emergenza specialmente in Finlandia, dove nacque la cosiddetta scuola finlandese, di indirizzo prettamente folcloristico, cui si deve la compilazione di importanti cataloghi, indici di tipi e motivi, in base ai quali è possibile tracciare, all'interno di un ambito storico-geografico ben definito, la diffusione di un determinato tipo fiabesco e le varianti, nonché le modificazioni da esso subite nel tempo e nello spazio. La classificazione proposta dalla scuola finlandese di Antti Aarne (The Types of the Folktale, 1961) fu completata e perfezionata dall'americano Stith Thompson, autore de La fiaba nella tradizione popolare (1946).

La fortuna dei Grimm in Italia non fu immediata come nel resto d'Europa. La più antica parziale edizione italiana delle fiabe grimmiane risale al 1875 ed è posteriore di quasi cinquant'anni a quella inglese (1823) e di quarantacinque a quella francese (1830). In Italia bisognerà attendere il 1908 per "disporre, a cura di un traduttore rimasto purtroppo anonimo, dell'edizione completa (Salani), smembrata però in cinque volumetti dai titoli casuali" ${ }^{[18]}$. Il ritardo della diffusione nella penisola italica dell'opera dei Grimm è dovuto al fatto che per molti e per molto tempo, la fiaba è stata oggetto di eccessive cautele pedagogiche. Esemplare a proposito dei Grimm la posizione assunta da Giuseppe Fanciulli (1881-1951), direttore di periodici fra cui «Il Corriere dei Ragazzi», curatore di biografie, di

[17] Calvino 1988a: 109.

[18] Cusatelli 1983: 123. 
studi e note di psicologia e di una storia della letteratura per l'infanzia scritta con Enrichetta Monaci Guidotti, La letteratura per l'infanzia. Se il Fanciulli riconosce ai due fratelli il merito di aver interpretato "l'anima germanica" attraverso "eterni temi" come "l'orgoglio umiliato, la malvagità invidiosa scoperta e punita, la bontà premiata dopo pene e guai", non manca altresì di rilevare nelle loro fiabe qualità negative "per il nostro gusto latino", come "l'abuso della crudeltà e della balordaggine", messe in evidenza con "particolari tragici e ripugnanti", dalle quali bisogna tener lontane "le giovani menti" [19]. Anche le certezze di Benedetto Croce (1866-1952) a proposito della fiaba hanno giocato un ruolo fondamentale nel far ignorare, almeno a livello di editoria fino al secondo dopoguerra, l'impegno della scuola finlandese, i repertori di motivi e tipi di Aarne e il fondamentale lavoro di Thompson che, uscito nel 1946, vede la luce in Italia solo vent'anni dopo, a trascurare i testi di Propp, il lavoro di Lüthi la cui opera, La fiaba popolare europea. Forma e natura, vedrà la luce in Italia più di trent'anni dopo la prima edizione tedesca (1947) ${ }^{[20]}$.

\section{L'indagine strutturale della fiaba di Propp, l'analisi stilistica di Lüthi e l'approccio psicoanalitico di Bettelheim}

Lo studio sistematico del genere fiabesco ha avuto un notevole contributo nello scorso secolo per merito della ricerca sulla fiaba condotta dello studioso russo Vladimir Jakovlevič Propp (1895-1970). Egli ha prodotto due opere ritenute fondamentali per la scientificità con cui sono state condotte le ricerche sulla struttura e le origini della fiaba. Dallo studio del patrimonio fiabistico russo, della raccolta ottocentesca di fiabe di Aleksandr Nikolajevič Afanasjev (1826-1871) in particolare, è nata la prima delle due opere, la Morfologia della fiaba (Morfologija skazki. Transformacij volshebnykh skazok), nella quale Propp individua un

[19] Fanciulli 1932: 22.

[20] Nel saggio Giambattista Basile e l'elaborazione artistica delle fiabe popolari, Croce scrive: "la questione dell'origine delle fiabe è da convertire ormai nella storia di ciascuna di esse, che è poi, a ogni suo passo, quella di una creazione a nuovo. Certo, sarebbe talvolta attraente seguire questa varia e intricata storia nei particolari; ma la cosa è assai difficile e mal sicura, trattandosi di processi fantastici che si svolgono quasi sempre fuori d'ogni osservazione e documentazione, e che ebbero forse il loro periodo intenso in tempi lontani, se non addirittura preistorici. I risultati, dunque, a cui questa parte si mette capo, di rado sono così concludenti da compensare la fatica; e poi, fatica o non fatica, hanno sempre piccola o niuna importanza". Il saggio di Croce, premesso all'edizione del Pentamerone ossia La fiaba delle fiabe di Giambattista Basile da lui curata nel 1925, è stato ripubblicato nel volume Il racconto dei racconti ovvero Il trattenimento dei piccoli curato da R. Guarini e A. Burani nel 1994, da cui è tratta la citazione crociana qui riportata (pp. 667-668). 
sistema di classificazione che permette di stabilire cos'è una fiaba prima di indagarne le origini. L'opera fu pubblicata in russo a Leningrado nel 1928 ma tradotta in lingua italiana con notevole ritardo solo nel 1966, anticipata non di molto dalla prima traduzione straniera (statunitense) del 1958 per opera di Laurence Scott (a cura e con introduzione di Svatava PirkovaJakobson), e acquistò notorietà più per il saggio introduttivo di Claude Lévi-Strauss, che ne sottolineava l'importanza, che per l'accuratezza dell'analisi effettuata da Propp sulle fiabe per definirne scientificamente le caratteristiche. Nonostante le iniziali difficoltà di divulgazione, l'opera ha avuto in seguito un'ampia risonanza, tanto che il metodo esposto dallo studioso russo è stato applicato all'indagine delle "leggi" della narratività. L'opera si pone difatti come punto di partenza degli studi sulla struttura del racconto, e, più in generale, del fatto artistico.

Analizzando cento fiabe prese dalla raccolta di Afanasjev (il campo d'indagine è limitato alla fiaba di magia russa) Propp ha scoperto che, pur avendo contenuti assolutamente diversi, tutti i racconti presentano una struttura monotipica: le azioni, definite funzioni (trentuno in tutto), che formano le parti fondamentali della fiaba ossia la base morfologica di tutte le fiabe di magia, sono elementi costanti, mentre la nomenclatura (re, principi, principesse, ecc.) e gli attributi (caratteristiche esteriori e psicologiche) dei personaggi variano ${ }^{[21]}$. Le funzioni, entro il cui limite si sviluppa la vicenda di tutte le fiabe prese in esame e la cui successione non cambia mai (il danneggiamento avviene prima della ricerca, l'apparizione del donatore precede il combattimento, il ritorno succede all'inseguimento), ricadono su sette personaggi: l'antagonista, il donatore, l'aiutante, il personaggio cercato, il mandante, l'eroe, il falso eroe. Questi personaggi sono soggetti di azione, cioè compiono l'azione o ne sono i protagonisti, e le loro funzioni non mutano nella fiaba, indipendentemente se essi sono tutti presenti o meno.

Dall'analisi proppiana si desume che lo schema compositivo della fiaba comprende una parte preparatoria (l'antefatto, che può anche mancare, prima dell'intervento dei personaggi) e la fiaba vera e propria che Propp definisce come "sviluppo da un danneggiamento (X) o da una

[21] A p. 7 della Morfologia della fiaba l'autore precisa: "Per attributi intendiamo l'insieme di tutte le caratteristiche esteriori dei personaggi: età, sesso, condizione, aspetto e suoi tratti particolari, ecc. Sono gli attributi a conferire alla fiaba la sua vivacità, la sua bellezza, il suo fascino". 
mancanza $(\mathrm{X})$ attraverso funzioni intermedie fino a un matrimonio $(\mathrm{N})$ o ad altre funzioni impiegate a mo' di scioglimento" ${ }^{[22]}$. Lo sviluppo della narrazione è chiamato movimento: ogni ulteriore danneggiamento dà origine a un nuovo movimento per cui, di conseguenza, ogni fiaba può constare di più movimenti. Ci possono essere raddoppi, triplicazioni, intrecci, deviazioni, ma lo schema di base resta sempre invariato. Lo schema è costituito dalla mancanza, dalla funzione intermedia e dallo scioglimento o risoluzione.

Al termine della sua rigorosa analisi, condotta su cento fiabe di magia, Propp afferma che esse hanno un'unica struttura, anzi, sostiene che rappresentano un'unica, grande fiaba universale. Con la sua ricerca, che è diventata un punto di riferimento ineludibile per ogni studio sulla fiaba, Propp ha stabilito la possibilità di fondare una sorta di grammatica del racconto, che tenesse conto dell'inventario di tutte le situazioni tipo che vi ricorrono in una cultura data e delle regole con cui esse si combinano. La moderna semiotica ha riconosciuto in Propp, sulla scorta di questa sua idea originale, uno dei suoi precursori. Notissimi autori dell'area strutturalista, tra cui Roland Barthes, Algirdas-Julien Greimas e Tzvetan Todorov, oltre al già citato Lévi-Strauss, hanno contribuito a diffondere l'idea di Propp in Occidente.

La seconda opera proppiana, Le radici storiche dei racconti di fate, una sorta di viaggio straordinario nel mondo dell'immaginario, mantiene intatto il suo potere evocativo anche a distanza di anni. Fu pubblicata in russo nel 1946, dopo quasi vent'anni dalla prima, di cui del resto costituisce una naturale prosecuzione poiché, secondo Propp, per definire le origini di un genere occorre acquisire prima una corretta conoscenza della sua struttura. Prima che nelle altre lingue europee, l'opera fu tradotta in lingua italiana già nel 1949. Nel 1972 ci fu un'altra traduzione italiana, di Salvatore Arcella, che corresse il titolo in Le radici storiche dei racconti di magia, sì da renderlo più aderente a quello originale, Istoričeskie korni volšebnoj skazki.

Ormai in possesso dei meccanismi di formazione del fiabesco, nell'opera Propp ha condotto uno studio diacronico sulla fiaba come fenomeno sovrastrutturale, ossia non dipendente dai rapporti sociali nell'ambito dei quali si manifesta. Diverse sono, infatti, le istituzioni

[22] Propp 1966: 98. 
storiche ed etnografiche a cui Propp fa ricorso per riferirle alla fiaba: egli spazia dalle credenze dei popoli primitivi della Malesia, dell'Africa e delle Americhe, ai rituali egizi assiri, greci. Attraverso uno studio storicogenetico che ricerca le origini della fiaba nei miti e nei rituali delle culture primitive, Propp conclude che le fiabe di magia conservano in sé tracce di riti tribali e d'iniziazione. L'idea non è del tutto nuova. Difatti già dalla pubblicazione dei Kinder und Hausmärchen dei Grimm l'esegesi scientifica della fiaba si era dedicata a questa caratteristica del racconto popolare: quella di essere cioè un immenso contenitore di immagini e simboli che si ritrovano sia nei miti e nei rituali di epoche passate sia nel mondo magicoreligioso dei popoli di interesse etnologico. L'origine delle fiabe non sarebbe il semplice risultato di vane fantasie, ma la decomposizione di miti antichissimi che risalgono all'età in cui i popoli interpretavano in maniera simbolica l'aurora, il tramonto, il sorgere delle stelle... In particolare, Propp collega la fiaba ai rituali d'iniziazione. L'iniziazione, presso i popoli primitivi, è un rito che si celebra al sorgere della pubertà, solitamente nella foresta o nel bosco, ove spesso i ragazzi vengono abbandonati dagli adulti. Tale rito, che permettere al ragazzo l'ingresso ufficiale nel mondo degli adulti, si compie ancora oggi in comunità particolarmente arcaiche. Presso alcune comunità dell'Oceania, per esempio, vige la credenza che i ragazzi da iniziare siano rapiti da un mostro divoratore. Così credono gli iniziandi fino alla notte del rito, quando scoprono che il mostro non esiste: si tratta degli anziani della comunità che con maschere e strumenti musicali imitano il ruggito del mostro. Anche questa acquisizione di "verità" fa parte della crescita. Tutto questo mondo simbolico del rituale (studiato ampiamente dagli etnologi) è rimasto parzialmente incapsulato in alcuni elementi narrativi del racconto fiabesco. L'esempio più evidente è la presenza, in molte fiabe, del motivo della cacciata dei bambini da casa, che vengono abbandonati a se stessi nel bosco, dove incontrano esseri terribili e minacciosi che essi devono sconfiggere per assurgere alla maturità ed essere accolti dalla comunità adulta.

All'analisi strutturale della fiaba proposta da Propp si affianca un'altrettanta puntuale indagine compiuta da Max Lüthi (1909-1991). L'importante studio di Lüthi, dedicato alla fenomenologia della fiaba, s'intitola La fiaba popolare europea. Forma e natura ed è stato pubblicato 
per la prima volta nel 1947. Attraverso un raffronto della fiaba popolare europea con altri generi consimili, soprattutto la leggenda profana e religiosa, lo studioso tedesco definisce le costanti fondamentali della fiaba. Esse sono: l'unidimensionalità, la mancanza di prospettiva, lo stile astratto, l'isolamento e le colleganze universali e, infine, la sublimazione e i contenuti universali. Dopo aver esaminato nel dettaglio ognuna delle costanti qui elencate, Lüthi conclude l'analisi soffermandosi sulla funzione e sul significato della fiaba che, secondo la sua tesi, "è una composizione poetica pura, non tendenziosa" ${ }^{[23]}$. In modo chiaro e diretto, "con gioiosa e leggera agilità la fiaba obbedisce a leggi severissime" [24], e realizza "quella coerenza stilistica che l'estetica moderna esige da qualsiasi vera opera d'arte" [25]. Contrariamente alla leggenda, che vuole spiegare e interpretare, la fiaba non esige nulla. Rinunciando a ogni spiegazione finalistica, la fiaba opta per una onirica rappresentazione del mondo. Essa non "interpreta o spiega nulla, limitandosi ad osservare e a rappresentare" ${ }^{[26]} \mathrm{e}$ a offrire una "risposta profondamente gratificante alle questioni scottanti dell'esistenza umana” ${ }^{[27]}$. Svuotando e sublimando, la fiaba trasfigura tutti i contenuti in "motivi fiabeschi", che nel perdere la loro concretezza e realtà guadagnano in trasparenza e determinatezza. La facoltà di sublimazione e la capacità di abbandonare una rappresentazione realistica e individualistica, permettono alla fiaba di accogliere contenuti universali, di aprirsi alla pienezza delle possibilità umane: il piccolo e il grande, eventi privati e collettivi, relazioni terrene e soprannaturali. Con la sua capacità di illuminare la vita rendendo trasparenti le ambiguità dei rapporti e le incertezze delle situazioni e indicando al contempo l'infinita ricchezza delle possibilità esistenziali, la fiaba rispecchia le componenti essenziali dell'esistenza umana. Il fatto che la comunità umana sia stata disposta a recepirla ed a coltivarla nel corso dei secoli, rispettando quelle caratteristiche che le sono autonomamente proprie, testimonia che essa soddisfa importanti esigenze umane, perché ciò che vive nella fiaba è il senso e il ricordo, forse inconsapevole, di millenarie esperienze collettive. Scrive Beatrice Solinas Donghi, sottolineando nello specifico la priorità del

[23] Lüthi 1982: 103.

[24] Lüthi 1982: 50.

[25] Lüthi 1982: 47.

[26] Lüthi 1982: 111.

[27] Lüthi 1982: 106. 
concetto estetico: "Se non fossero state belle e se non fossero sembrate belle di generazione in generazione, né Afanasjev né Propp le avrebbero raccolte. Una fiaba orale se non sta insieme tanto da poter essere ricordata, non può sopravvivere" ${ }^{[28]}$. Lo stile astratto della fiaba, che consente di caricare di significati personali e valenze emotive il contesto e i personaggi fiabeschi, coinvolgendo non solo la sfera razionale ma anche quella emozionale dell'ascoltatore o del lettore, nonché la sua grande carica magica, fanno di questo genere letterario un prezioso veicolo per un avvio alla lettura finalizzato esclusivamente al piacere.

A Bruno Bettelheim (1903-1990), psichiatra e psicoanalista statunitense di origine austriaca, studioso di psicologia sociale e di psicosi infantile, in particolare dell'autismo, si deve una delle più illuminanti chiavi interpretative della fiaba, quella psicoanalitica, secondo la quale la fiaba è la sola forma narrativa idonea a rispondere ai bisogni interiori del bambino. Partendo dalla teoria dello psicologo svizzero Jean Piaget (18961980), secondo la quale il bambino è animistico fino all'età della pubertà (La rappresentazione del mondo nel fanciullo, 1936), Bettelheim desume che la fiaba si adegua in modo ideale alla sua mentalità e che l'elemento magico, tipico del fiabesco, è in grado di aiutare il fanciullo ad affrontare e risolvere i problemi dello sviluppo ${ }^{[29]}$. L'autore afferma che le storie realistiche sono estranee al modo di funzionare della mente del bambino e non gli dicono nulla sulle sue esperienze interiori: esse lo informano senza arricchirlo. Se al bambino, oltre alle storie realistiche, vengono raccontate anche le fiabe, egli riceve informazioni che riguardano sia l'aspetto razionale sia quello emotivo della sua personalità. Ne Il mondo incantato. Uso, importanza e significati psicoanalitici delle fiabe Bruno Bettelheim scrive: "Chiaramente la fiaba non si riferisce al mondo esterno, anche se può iniziare in modo abbastanza realistico e avere, intessuti in essa, elementi della vita di tutti i giorni" [30]. In verità, secondo lo studioso, la natura realistica della fiaba rappresenta "un importante espediente, perché evidenzia che il proposito di una fiaba non è quello di comunicare utili

[28] Solinas Donghi 1976: 172.

[29] Esiste una fase nella vita del bambino che è "la fase della fiaba", compresa tra i quattro e i sette anni. In questa fase la visione del mondo della fiaba coincide con quella del bambino. In questo periodo scompaiono i confini tra realtà e fantasia per cui il bambino oscilla con assoluta naturalezza dal piano reale a quello fantastico: vive la realtà in forma magico-animistica e, come nelle fiabe, dà vita agli oggetti e attribuisce loro particolari poteri e qualità umane.

[30] Bettelheim 1977: 29. 
informazioni circa il mondo esterno, ma di chiarire i processi interiori che hanno luogo in un individuo" [31]. Perché, come spiega ancora Bettelheim nel suo interessante lavoro, nel "contenuto delle fiabe vengono espressi in forma simbolica fenomeni psicologici interiori” [32].

Bettelheim ha indubbiamente delle acute intuizioni quando dal punto di vista psicoanalitico pone in rilievo che le fiabe popolari, in quanto sedimentazione storica di profonde esperienze umane, hanno una funzione terapeutica per il bambino, scaricando sue paure e frustrazioni come la paura di venir abbandonato, di non sentirsi considerato e amato, oppure delusioni narcisistiche, rivalità fraterne, gelosie parentali, dilemmi edipici, ecc. Queste storie parlano "delle sue gravi pressioni interiori in un modo che il bambino inconsciamente comprende, e - senza sminuire le violente lotte interiori che il processo di crescita comporta - offrono esempi di soluzioni sia permanenti sia temporanee a pressanti difficoltà" ${ }^{[33]}$. E prosegue: "la forma e la struttura delle fiabe suggeriscono al bambino immagini per mezzo delle quali egli può strutturare i propri sogni ad occhi aperti e con essi dare una migliore direzione alla sua vita" ${ }^{[34]}$. Le fiabe sono maggiormente significative per il bambino perché nell'età in cui si trova “il suo problema principale è quello di mettere un po' d'ordine nel caos interiore della sua mente per poter capirsi meglio: un preliminare necessario per il conseguimento di una certa congruenza fra le sue percezioni e il mondo esterno" ${ }^{[35]}$. A livello intuitivo il bambino capisce che queste storie, sebbene irreali, non sono false: il bambino sa che quanto viene narrato nelle fiabe non accade nella realtà, ma avviene come esperienza interiore e sviluppo personale. "Il bambino cerca nella fiaba quasi la conferma di ciò che in un certo senso egli sa già con la sua logica e la sua verità" [36], osserva Rita D'Amelio. Si può pertanto parlare a ragione d'isomorfismo fra la struttura logica infantile e la struttura logica della fiaba. A livello simbolico e in forma immaginaria la fiaba descrive le tappe fondamentali del processo di sviluppo, teso all'acquisizione di un'esistenza indipendente. Siccome il pensiero del bambino è essenzialmente dicotomico e incapace di coglier le sfumature e i molteplici risvolti della condotta umana, dal punto di vista

[31] Bettelheim 1977: 29.

[32] Bettelheim 1977: 39.

[33] Bettelheim 1977: 12

[34] Bettelheim 1977: 13

[35] Bettelheim 1977: 55.

[36] D’Amelio 1979: 19. 
morale il bambino si pone fin dall'inizio, come osserva Bettelheim, al posto giusto: si sente naturalmente attratto dalla figura dell'eroe e vi si identifica scegliendo in modo inequivocabile il bene. In tal senso la fiaba favorisce nel bambino la formazione di una coscienza morale. E siccome l'ethos della fiaba è una moralità "primitiva", pre-esistente ai condizionamenti socioculturali delle civiltà sviluppate, questa moralità è trasparente, pura: uno specchio in cui l'uomo (e il bambino) vede riflesse le sue azioni, così come sono e come dovrebbero essere. Non c'è contrapposizione tra reale e ideale bensì identità. Come spiega Lüthi nell'opera sopra citata, "la fiaba non ci mostra un mondo in ordine, ci mostra il mondo in ordine. Ci mostra che il mondo è così come dovrebbe essere" [37].

Per anticipare chi potrebbe osservare che le fiabe appartengono a un'epoca troppo remota e non propongono pertanto contenuti adeguati alla nostra contemporaneità, Bettelheim precisa: "Certo, a livello manifesto le fiabe hanno poco da insegnare circa le specifiche condizioni di vita nella moderna società di massa; queste storie furono create molto tempo prima del suo avvento. Ma esse possono essere più istruttive e rivelatrici circa i problemi interiori degli esseri umani e le giuste soluzioni alle loro difficoltà in qualsiasi società, di qualsiasi altro tipo di storia alla portata della comprensione del bambino" ${ }^{[38]}$. Ma perché la fiaba possa realizzare compiutamente la sua valenza educativa e "comunicare appieno i suoi messaggi consolatori, i suoi significati, simboli e, soprattutto, i suoi significati interpersonali", essa "dovrebbe essere raccontata piuttosto che letta", perché la "narrazione della storia a un bambino, per ottenere la massima efficacia, deve essere un fatto interpersonale, plasmato da coloro che vi partecipano" [39]. Oltre alla valenza educativa e formativa della fiaba, l'affermazione di Bettelheim mette in rilievo l'importanza dell'oralità, che ha contrassegnato la fiaba sin dalla sua origine, e il significato del profondo rapporto affettivo, di complicità, di empatia e di confidenza che si stabilisce tra chi narra e chi ascolta. La narrazione di una fiaba dovrebbe essere difatti un'esperienza emotiva condivisa, un evento plasmato sia dall'adulto sia dal bambino ${ }^{[40]}$. Nel suo ragionamento Bettelheim è in perfetta sintonia

[37] Lüthi 1982: 111.

[38] Bettelheim 1977: 11.

[39] Bettelheim 1977: 147-148.

[40] Il modo esemplare in cui si dovrebbe raccontare una fiaba è quello applicato per il figlio dalla madre di Goethe, che in vita fu nota come una grande narratrice di fiabe, come ricorda Bettelheim a pp. 149-150 dell'opera qui citata, in cui riporta un brano tratto dal memoriale scritto dall'ormai anziana madre dello 
con l'atteggiamento verso la fiaba dichiarato in più occasioni da Calvino e da Rodari. Entrambi hanno esaltato il valore morale e formativo del genere fiabico, ritenendolo la forma narrativa principe per il bambino e un dono prezioso che l'umanità ha fatto a se stessa ${ }^{[41]}$. Da Bettelheim ci giunge ancora un ultimo prezioso suggerimento: il significato intrinseco e pedagogico della fiaba non deve essere mai svelato al bambino. Egli deve rimanere inconsapevole delle pressioni inconsce, fare proprie soltanto le sensazioni offerte dal racconto e cogliere solo quei significati della fiaba che lo interessano nel preciso momento in cui l'adulto gliela racconta. Solo più in là nel tempo, se e quando lo vorrà, potrà scoprire da solo nella fiaba anche altri significati. Bettelheim descrive in modo simbolico questo processo: "Ascoltare una fiaba e recepire le immagini che essa presenta può essere paragonato a uno spargimento di semi, che solo in parte germogliano nella mente del bambino. Alcuni di essi hanno immediatamente effetto nella sua mente; altri stimolano processi nel suo inconscio. Altri ancora hanno bisogno di riposare a lungo fino a che la mente del bambino abbia raggiunto uno stadio idoneo alla loro germinazione, e molti non metteranno mai radici. Ma quei semi che sono caduti sul terreno adatto produrranno fiori meravigliosi e alberi gagliardi - cioè daranno validità a importanti sentimenti, incoraggeranno intuizioni, nutriranno speranze, ridurranno ansie - e così facendo arricchiranno la vita del bambino nel presente e per il resto della sua vita" ${ }^{[42]}$.

Allo studio di Bettelheim, che con i suoi collaboratori presso la "Orthogenic School" per i bambini psicotici di Chicago ha utilizzato la fiaba in funzione diagnostica e terapeutica, ha fatto seguito la pubblicazione di

scrittore: "Inventammo strade tra le stelle, e fantasticammo sulle grandi menti che ivi avremmo incontrato... Egli mi divorava con gli occhi, e se la sorte di una delle sue favorite non corrispondeva ai suoi desideri potevo avvedermene dalla collera dipinta sul suo volto, o dai suoi sforzi per non scoppiare in lacrime. Di quando in quando interveniva col dire: "Madre, la principessa non sposerà il suo miserabile sarto, neppure se uccide il gigante", al che io mi arrestavo e rimandavo la catastrofe alla sera seguente. Così la sua immaginazione prese sovente il posto della mia, e quando la mattina dopo correggevo il fatto secondo i suoi suggerimenti e dicevo: "Avevi indovinato, fu così che andò a finire", era tutto eccitato, e si poteva vedere il suo cuore che batteva". Il modo in cui la madre di Goethe raccontava le fiabe a suo figlio è descritto da Elizabeth (Bettina) von Arnim in Goethe's Briefwechsel mit einem Kinde (1906), in traduzione italiana Il carteggio di Goethe con una bimba (Treves, Milano, 1932).

[41] Negli anni Cinquanta dello scorso secolo, all'inizio della sua fortunata carriera di scrittore per l'infanzia, Gianni Rodari ha preso coscienza del valore storico-narrativo della fiaba e l'ha acquisita come canovaccio del suo lavoro letterario in prosa. In un lavoro del 1970, Pro e contro la fiaba, ha evidenziato che le fiabe sono "un deposito stratificato di più culture, un archivio in cui il tempo ha depositato le sue pratiche", un "relitto di cui la fantasia popolare ha fatto il suo tema" (Gianni Rodari, in Il cane di Magonza, Editori Riuniti, Roma, 1982, p. 143). Per Rodari il fiabista-tipo è Andersen. Nei testi anderseniani, l'uso creativo della fiaba, la ripresa del fiabico attraverso affabulazioni originali e d'«autore», sono considerati come il contrassegno più evidente e prezioso. Andersen è il creatore di quella fiaba contemporanea di cui anche Rodari è, e vuole essere, l'erede.

[42] Bettelheim 1977: 151. 
tutta una serie di lavori che affrontano la fiaba partendo dalla concezione psicoanalitica ${ }^{[43]}$. La sua ricerca però, oltre ad aver rivoluzionato le teorie al momento consolidate intorno alla valenza psicologica e educativa del materiale fiabesco, è anche la più didatticamente profusa ${ }^{[44]}$. L'opera del grande psicologo austriaco, attraverso una limpida riflessione e per mezzo di acute e suggestive analisi di numerose fiabe, tra le più note e amate, da Cappuccetto Rosso a Cenerentola, da Biancaneve a Hänsel e Gretel, non solo presenta in modo affascinante e insolito questo genere di narrativa, ma programma una precisa strategia educativa di un suo uso pedagogico concreto e corretto. Nella sua opera Bettelheim avverte che la fiaba si adegua in modo ideale alla mentalità del bambino, rimasta in fondo immutata attraverso i secoli. Pertanto, lungi dall'essere sorpassata, rispecchiando la visione magica e animistica che il bambino ha delle cose, i suoi stupori, i suoi terrori, i suoi desideri impossibili, la fiaba esorcizza incubi sepolti nell'inconscio, placa inquietudini, aiuta a superare crisi e incertezze esistenziali, ad accettare responsabilità, e mette il bambino di fronte alle reali difficoltà dell'esistenza con un linguaggio non realistico, che è poi l'unico da lui pienamente recepibile a livello profondo. Collocandosi al di fuori delle categorie dell'ovvio e del convenuto, oltre ad essere per il bambino un'autentica introduzione alla vita, la fiaba è anche "il luogo di tutte le ipotesi [...] la chiave per entrare nella realtà per strade nuove" [45] e, quindi, anche un potente stimolo alla creatività e al pensiero divergente.

\section{Le Fiabe italiane di Italo Calvino}

Al recupero del patrimonio fiabesco tradizionale, contestualmente alla rielaborazione di miti e fiabe di origine etnico-popolare, si applicarono in Italia intelligenti curatori di raccolte di fiabe, popolari e d'autore, che le

[43] Richiamandosi a Bettelheim, Anna Calò in Il mondo della fiaba e i bisogni psicologici del bambino (Mirella, Lecce, 1981) presenta una esauriente e lucida analisi che dimostra come la fiaba corrisponda ai bisogni psicologici del bambino (bisogno di integrazione, di indipendenza e autonomia, bisogno di costruirsi un linguaggio proprio e propri schemi concettuali) e contribuisca a risolvere i conflitti delle fasi orale, anale e fallica e i complessi che si instaurano nell'ambito familiare.

[44] Gli psicanalisti freudiani indagano sul materiale inconscio che sta alla base delle fiabe e si preoccupano di mostrare il loro rapporto con $i$ sogni, le fantasie e gli avvenimenti interiori dell'uomo. Per gli psicanalisti junghiani le fiabe sono l'espressione dei processi psichici dell'inconscio collettivo e rappresentano gli archetipi, immagini che appartengono alla storia dell'umanità, in forma semplice e concisa. Un ricco contributo all'interpretazione secondo la scuola junghiana è stato dato da Marie Louise von Franz nei volumi: Le fiabe interpretate, Il femminile nella fiaba, L'individuazione nella fiaba, Le fiabe del lieto fine.

[45] Rodari 1973: 140-141. 
raccolsero con viva sensibilità estetica e pedagogica: Mary Tibaldi Chiesa (Meravigliose fiabe italiane, 1960), Lino Moncherini (C'era una volta, 1968), Guido Davico Bonino (Le più belle fiabe italiane, 1987) e Francesca Lazzarato, colta saggista e finissima traduttrice (I sette libri delle fiabe, 1987-1989). Ė stato però Italo Calvino che, per primo e con superiore sensibilità artistica, su iniziativa della Casa editrice Einaudi presso la quale era stato assunto come redattore stabile nel gennaio 1950, si impegnò a raccogliere sistematicamente il patrimonio fiabistico italiano, e lo fissò per sempre nella fondamentale raccolta intitolata Fiabe italiane, pubblicata nel 1956. Stranamente, se si considera che i grandi libri di fiabe sono nati in Italia in anticipo rispetto ad altri paesi europei, fino a quel momento l'Italia non aveva ancora avuto il "suo Grimm" ${ }^{46]}$. C'era una mole di materiale, una montagna di narrazioni tratte dalla bocca del popolo nei vari dialetti, raccolte da studiosi come Angelo De Gubernatis, Vittorio Imbriani, Domenico Comparetti e Giuseppe Pitré, ma era un patrimonio destinato a fermarsi nelle biblioteche degli specialisti, a essere argomento di studio dei "demopsicologi", come si chiamarono per un certo tempo con un termine coniato dal Pitré gli studiosi del folclore, non a circolare in mezzo al pubblico. Era giunto il momento, anche per l'Italia, di impossessarsi di quella parte fondamentale della sua tradizione popolare. Le Fiabe italiane nascono, a detta del curatore, con lo scopo di "ravvivare in Italia un interesse per queste ricerche [sulla fiaba]", e perché "tra i nostri studi sul folklore tornino ad avere il posto dovuto quelli sulla novellistica popolare, e le lacune esistenti per molte ragioni vengano colmate, e soprattutto siano ricerche intelligenti, aggiornate sulle esperienze straniere più nuove $\mathrm{e}$ interessanti compiute in questo campo" ${ }^{[47]}$.

Autore di originali fiabe moderne di tono paradossale e grottesco, percorse da una sottile vena caustica e corrosiva, contestativa degli aspetti più brutalmente alieni della civiltà tecnologica, adatte a un lettore adolescente (Il barone rampante, 1959; Marcovaldo ovvero le stagioni

[46] Già nella metà del Cinquecento a Venezia, in Le piacevoli notti di Francesco Straparola, la novella cede il posto alla fiaba di magia. Nel Seicento barocco, a Napoli, Giambattista Basile scrive in dialetto napoletano e con gusto esplicito per il meraviglioso, conforme all'epoca, il Pentamerone o Lo cunto de li cunti. Ancora a Venezia, nel Settecento illuminista, Carlo Gozzi, astioso e conservatore, contestatore della riforma goldoniana del teatro, fa calcare alle fiabe le tavole del palcoscenico, tra le maschere della commedia dell'arte. Nel secondo Ottocento positivista, Luigi Capuana scrive C'era una volta..., libro di fiabe nutrite insieme di fantasia e di spirito popolare.

[47] Calvino 1988a: 22. 
in città, 1963; Il visconte dimezzato, 1972), con le Fiabe italiane, che rappresenta la prima raccolta nazionale di fiabe popolari italiane nella lingua standard ${ }^{[48]}$, Calvino ha reso omaggio alla "scientificità a metà" dei fratelli Grimm, ai quali può essere accostato specialmente per il suo lavoro di riscrittore ${ }^{[49]}$. Pur se non destinato agli specialisti bensì a un pubblico eterogeneo e privo di una conoscenza di base sulla fiaba e sul folclore, il lavoro di Calvino è importante dal punto di vista documentario, e anticipa quell'interesse per la fiaba che sarebbe stato più tardi al centro delle ricerche della narratologia, come auspicava nel passo sopra citato, specialmente in seguito alla pubblicazione della Morfologia della fiaba di Propp. Benché costituisca un'operazione culturale di capitale importanza, l'opera di Calvino, a differenza del profondo effetto di quella dei Grimm nella società tedesca dell'Ottocento, non ha destato in Italia lo stesso interesse e l'attenzione che avrebbe meritato. È arrivata in ritardo, anacronisticamente in un'epoca di scarsa traduzione orale e in una società nella quale, pur se l'infanzia andava sempre più alfabetizzandosi, predominavano già altre fonti dell'immaginario infantile.

La scelta della Casa editrice Einaudi di affidare nel 1954 a Calvino questo delicato e complesso lavoro non è stata casuale, se si considera che lo scrittore, come aveva notato per primo Cesare Pavese, aveva una predisposizione innata per il fiabesco, manifestatasi già all'inizio della sua fortunata carriera di scrittore in Il sentiero dei nidi di ragno, il suo primo romanzo, e nei primi racconti scritti nel 1946 e 1947. Nel caso di Calvino si potrebbe dire che il fiabesco stava scritto nel suo destino. Infatti, già ne Il sentiero dei nidi di ragno del 1947, incentrato sulla Resistenza, al momento di avvicinarsi in pieno neorealismo a questa "materia epica" per riprenderla e rielaborarla, Calvino si era servito degli occhi di un ragazzo, Pin, il protagonista, che "da un lato gli avevano permesso di evitare le insidie della retorica, sottoponendo la realtà a un fortissimo

[48] Non va dimenticato che già nel Seicento Giambattista Basile (1575-1632) aveva scritto una prima raccolta di fiabe, ma le aveva scritte in dialetto napoletano, il Pentamerone o Lo cunto de li cunti. La raccolta di Basile consta di cinquanta racconti realistico-fiabeschi, che si fingono raccontati in cinque giornate da dieci vecchie, caratterizzati dal gusto barocco per la metafora e da una straordinaria vivacità espressiva, tipica del dialetto napoletano. Lopera calviniana è la prima raccolta che fissa in lingua standard il patrimonio fiabistico italiano.

[49] Nell'opera Sulla fiaba, curata da Mario Lavagetto, a p.20, nel saggio Le fiabe italiane, nella parte intitolata Criteri del mio lavoro, Calvino scrive: "Questo richiamo [ai fratelli Grimm] serve a introdurre e giustificare (facendomi scudo di nomi così famosi e così distanti) la natura ibrida del mio lavoro, che è anch'esso «scientifico» a metà, o se vogliamo per tre quarti, e per l'ultimo quarto frutto d'arbitrio individuale". 
straniamento, e dall'altro lo avevano portato ad avvicinarsi alle cose con lo sguardo di chi scopre il mondo per la prima volta e ritrova, dietro le apparenze, lo stampo delle favole più remote, «lo schema insostituibile di tutte le storie umane»" ${ }^{50]}$. Pur se maturato in fretta e in mezzo alla strada e alla violenza, Pin ha ancora stupori e malinconie e può inserirsi nelle vicende degli adulti con una disposizione fantastica e avventurosa che carica, e così trasfigura, quelle vicende, appunto perché a lui non del tutto comprensibili, di un alone fiabesco. Due anni prima di dedicarsi alle Fiabe italiane, Calvino aveva già scritto Il visconte dimezzato (1952), incluso più tardi con Il barone rampante (1957) e Il cavaliere inesistente (1959) nella trilogia I nostri antenati (1960). È un racconto dove il fiabesco è fortissimo e la narrazione ruota intorno all'immagine di un visconte tagliato in due metà durante uno scontro con i Turchi, una buona (il Buono) e una cattiva (il Gramo), che vivono scisse indipendentemente e malamente, finché alla fine non si ricompongono in un essere unico, costituito dall'intreccio di entrambe le parti. Il tema è quello tipicamente fiabesco dell'eterno conflitto tra bene e male, rappresentato nella fiaba sempre nitidamente e senza ambiguità. Le due parti del visconte, opposte e complementari, rappresentano ciascuna un aspetto parziale dell'umanità. Vivono svariate avventure prima di ricomporsi in un'unica persona, Medardo, nel quale il bene e il male troveranno il giusto equilibrio. Solo così egli diventerà onesto e retto governatore delle sue proprietà.

Le fiabe raccolte da Calvino sono duecento, in conformità con la raccolta dei Grimm, ricavate dalle più diverse tradizioni regionali e trascritte in una lingua semplice e piana, che riduce ai minimi termini "connotazioni stilistiche troppo marcate, per rivolgersi a una "media" comunicazione nazionale, accessibile anche ai bambini" [51]. L'arte di Calvino si manifesta proprio nella perfezione dello stile, che resta nel solco dell'espressione popolare e valorizza pertanto i pregi delle fonti. Lo scrittore è un narratore discreto, soltanto un anello dell'anonima catena senza fine per cui le fiabe si tramandano. Solo così, con un continuo e minuzioso controllo dei registri delle voci dei narratori che gli si affollano intorno, capace di neutralizzare da un lato le differenze dei dialetti o le deformazioni dei raccoglitori e dall'altro le proprie resistenze culturali, Calvino riesce a

[50] Così Mario Lavagetto nell'Introduzione a Sulla fiaba, p. IX.

[51] Ferroni 2007: 144 . 
salvaguardare "il «diverso» che proviene dal modo di raccontare del luogo e dall'accento personale del narratore orale" ${ }^{[52]}$, e a riprodurre un mondo che, nella sua molteplicità, appare di straordinaria "ricchezza e limpidezza e variegatezza" ${ }^{[53]}$. Nel passo che riportiamo di seguito lo scrittore riassume il metodo con cui ha lavorato, che non si discosta da quello applicato dai Grimm: si trattava, spiega Calvino, di "scegliere da questa montagna di narrazioni [...] le versioni più belle, originali e rare; tradurle dai dialetti in cui erano state raccolte [...]: arricchire sulla scorta delle varianti la versione scelta [...]; integrare con mano leggera d'invenzione i punti che paiono elisi o smozzicati; tener tutto sul piano d'un italiano mai troppo personale e mai troppo sbiadito, che per quanto possibile affondi le radici nel dialetto, senza sbalzi nelle espressioni «colte», e sia elastico abbastanza per accogliere e incorporare dal dialetto le immagini, i giri di frase più espressivi e inconsueti" [54]. Calvino precisa ancora che "variano da fiaba a fiaba la misura e la qualità del mio intervento, a seconda di quel che il testo mi suggeriva. Talora esso m'imponeva un rispetto che non potevo che cercare di tradurlo pari pari [...], talaltra invece mi si presentava come un mero punto di partenza per un esercizio di stile" [55]. Seguendo un criterio di gusto e sensibilità che si è autoimposto, scegliendo "la strada di un recupero sofisticato e consapevolmente letterario da risultare pressoché impercettibile", Calvino "non lascia tracce apparenti del proprio intervento anche quando questo intervento - come dimostra un esame dei palinsesti - è cospicuo" [56]. Perciò può concludere: "Ho inteso di mettermi anch'io come un anello dell'anonima catena senza fine per cui le fiabe si tramandano, anelli che non sono mai puri strumenti, trasmettitori passivi, ma [...] i suoi veri «autori»" ${ }^{[57]}$. Perché, nel passare di bocca in bocca, la fiaba ha naturalmente subito modifiche, in quanto il narratore, nel momento stesso in cui crede di rispettare scrupolosamente il modello e lo scheletro che ha tra le mani, sfugge alle maglie di una ripetizione puramente passiva con una istintiva furberia e finisce per parlarci di quello che gli sta a cuore. Sulla superficie del testo restano e si sovrappongono le impronte digitali delle mani attraverso cui, nella sua secolare esistenza, il racconto

[52] Calvino 1988a: 27

[53] Calvino 1988a: 17.

[54] Calvino 1988a: 21.

[55] Calvino 1988a: 27-28.

[56] Così Mario Lavagetto, in Introduzione a Sulla fiaba, p. XVI.

[57] Calvino 1988a: 28. 
è passato: all'interesse per queste impronte, che vengono attentamente registrate e conservate nella trascrizione il più possibile, si ricollega in Calvino l'interesse per la figura dei novellatori e delle novellatrici, lo stesso interesse che verso i loro "informatori" manifestarono i fratelli Grimm. Anche Calvino, come i Grimm, ha la sua narratrice privilegiata nella figura della siciliana Agatuzza Messia, la nutrice di Giuseppe Pitré ${ }^{[58]}$, alla quale si devono alcune delle narrazioni più perfette e compiute. Calvino le riconosce fantasia, precisione, competenza narrativa, controllo perfetto dei meccanismi, sorprendente abilità descrittiva e nomenclatoria. Agatuzza Messia rappresenta per Calvino quello che "frau Viehmännin" era stata per i fratelli Grimm: il modello della novellatrice ideale, nella cui voce la fiaba prende corpo e fisionomia. Non stupisce pertanto che la Sicilia sia la regione che ha offerto a Calvino il materiale più copioso e affascinante. In Le fiabe italiane, il saggio inserito da Mario Lavagetto nell'opera Sulla fiaba, che comprende in volume gli scritti di Calvino sul genere fiabesco, nel capitolo intitolato Le raccolte folkloristiche, lo scrittore riporta un passo tratto dalla prefazione della raccolta di fiabe di Giuseppe Pitré, dove il folclorista siciliano descrive dettagliatamente Agatuzza Messia, la sua nutrice e narratrice modello: "Tutt'altro che bella, essa ha la parola facile, frase efficace, maniera attraente di raccontare, che ti fa indovinare della sua straordinaria memoria e dell'ingegno che sortì da natura. [...] La Messia non sa leggere, ma la Messia sa tante cose che non le sa nessuno, e le ripete con una proprietà di lingua che è un piacere a sentirla. [...] La Messia mi vide nascere e mi ebbe tra le braccia: ecco perché io ho potuto raccogliere dalla sua bocca le molte e belle tradizioni che escono col suo nome. Ella ha ripetuto al giovane le storielle che aveva raccontate al bambino di trenta anni fa; né la sua narrazione ha perduta un'ombra della antica schiettezza, disinvoltura e leggiadria" ${ }^{[59]}$. Come sottolinea di seguito il Pitré nell'interessante prefazione, nella sua narrazione la Messia

[58] Nella seconda metà del XIX secolo, gli studi di folclore che in Inghilterra, in Francia e in Germania si erano dati statuto di scienza, e che avevano trovato in Italia patroni della statura d'un D'Ancona e d'un Comparetti, ebbero la loro epoca d'oro in Sicilia. Le raccolte di Lionardo Vigo (Canti popolari siciliani, 1857) e di Salvatore Salomone Marino (Canti popolari siciliani, 1867), aprirono la strada al lavoro di Giuseppe Pitré (1841-1916), un medico votatosi agli studi di folclore, autore di Fiabe, novelle e racconti popolari siciliani, quattro volumi che contengono trecento narrazioni (e cento varianti riassunte in nota) di tutte le province della Sicilia, e fondatore della Biblioteca delle tradizioni popolari siciliane (25 voll., 1870-1913). Fu in contatto con i maggiori cultori europei del folclore e raccolse una fondamentale Bibliografia delle tradizioni popolari d'Italia (1894). Fondò a Palermo il Museo etnografico a lui intitolato.

[59] Calvino 1988a: 32. 
trae spunto dalla natura, sollecita il meraviglioso ma lo fa nascere da un dato realistico, da una rappresentazione della condizione del popolo. La sua lingua è ricca d'invenzione ma pur sempre ancorata ai modi di dire e al buon senso popolari, "a immagini tutte prese dagli agenti esterni, per le quali diventano concrete le cose astratte, corporee le soprasensibili, vive e parlanti quelle che non ebbero mai vita o l'ebbero solo una volta" [60].

Durante l'arduo lavoro preparatorio, Calvino si è avvalso dell'assistenza dell'etnologo Giuseppe Cocchiara, ispiratore, per la collana dei «Millenni» dell'Einaudi, della collezione dei «Classici della fiaba». Ha lavorato su materiale già raccolto e pubblicato in libri e riviste specializzate, oppure reperibile in musei o biblioteche. Aveva a disposizione una gran mole di materiale, raccolto in gran parte dai folcloristi dell'Ottocento. Nel titolo le fiabe sono definite "italiane" non perché siano esclusive e originarie dell'Italia, ma semplicemente perché fanno parte del patrimonio fiabistico del folclore italiano, raccolto dalla viva voce del popolo. Del resto, come abbiamo già evidenziato in questo contributo, Calvino considerava che fosse inutile collocare nello spazio e nel tempo le fiabe, che sono uguali dappertutto, per cui dire "di dove" una fiaba sia non ha molto senso. Le fiabe, insomma, sono di natura migratoria: viaggiano nello spazio e nel tempo, attraversano secoli e continenti, ma attraversano anche strati sociali, descrivendo di volta in volta un percorso di ascesa o di discesa. Così Calvino spiega la scelta del titolo: "Diciamo dunque italiane queste fiabe in quanto raccontate dal popolo in Italia, entrate per tradizione orale a far parte del nostro folklore narrativo e similmente le diciamo veneziane o toscane o siciliane; e poiché la fiaba, qualunque origine abbia, è soggetta ad assorbire qualcosa dal luogo in cui è narrata - un paesaggio, un costume, una moralità, o pur solo un vaghissimo accento o sapore di quel paese - , il grado in cui si sono imbevute di questo qualcosa veneziano o toscano o siciliano è appunto il criterio preferenziale della mia scelta" ${ }^{\text {[61] }}$.

E interessante rilevare infine quanto Calvino scrive a proposito delle caratteristiche delle fiabe italiane, se paragonate a quelle tedesche. Riportiamo il suo giudizio: "La naturale «barbarie» della fiaba si piega [nella fiaba italiana] ad una legge d'armonia. Non c'è qui quel continuo informe schizzar di sangue dei crudeli Grimm; è raro che la fiaba italiana raggiunga

[60] Calvino 1988a: 33.

[61] Calvino 1988a: 24. 
la truculenza, e se è continuo il senso della crudeltà, dell'ingiustizia anche disumana, come elemento con cui si devono sempre fare i conti, se i boschi echeggiano anche qui dei pianti di tante fanciulle o spose abbandonate con le mani mozze, la ferocia sanguinaria non è mai gratuita, e la narrazione non si sofferma ad infierire sulla vittima, neppure con un'affermazione di pietà, ma corre verso la soluzione riparatrice" ${ }^{[62]}$. Considerando che le fiabe potranno avere come fruitori anche i bambini, Calvino conclude: "Nelle mie stesure, per le quali ho dovuto tener conto dei bambini che le leggeranno o a cui saranno lette, ho naturalmente smorzato ogni carica di questo genere" [63].

Calvino è il curatore della raccolta, autore solo in quanto ha organizzato, rielaborato e trascritto le fiabe. Prima del lavoro eseguito da Calvino l'Italia non possedeva una raccolta nazionale di fiabe popolari in lingua, per cui l'opera non ha precedenti nella letteratura italiana. Può essere paragonata per il suo valore culturale solo a capolavori europei della stessa portata, alla raccolta dei fratelli Grimm, a quella di Afanasjev, di Perrault e alle fiabe del danese Andersen, che si distacca però dalla tradizione orale per creare racconti fantastici in cui dà voce alla propria memoria e alla propria vicenda biografica piuttosto che a quella del suo popolo. Nel momento in cui ha terminato un lavoro di cui aveva colto tutta la complessità sin da quando gli era stato affidato, quando "il viaggio tra le fiabe è finito" e "il libro è fatto", Calvino si pone una domanda che ci pare ovvia: "[...] riuscirò a rimettere i piedi sulla terra? Per due anni ho vissuto in mezzo a boschi e palazzi incantati [...] E per questi due anni a poco a poco il mondo intorno a me veniva atteggiandosi a quel clima, a quella logica, ogni fatto si prestava a essere interpretato e risolto in termini di metamorfosi e incantesimo [...] Ogni poco mi pareva che dalla scatola magica che avevo aperto, la perduta logica che governa il mondo delle fiabe si fosse scatenata, ritornando a dominare la terra" ${ }^{[64]}$. Se prendiamo le parole dello scrittore alla lettera, ci rendiamo conto che il suo non è soltanto un modo di dire: le sue parole esprimono, in forma metaforica, un problema reale, di scrittura. «Rimettere i piedi in terra»: è un problema al quale una soluzione deve essere trovata. E Calvino la troverà perché,

[62] Calvino 1988a: 48-49.

[63] Calvino 1988a: 51.

[64] Calvino 1988a: 18. 
esaurita la risorsa del fiabesco, che non abbandonerà mai del tutto, cercherà sussidi alla sua vena fantastica nei suggerimenti che gli derivano dalla scienza, dalla filosofia, dalla teoria letteraria. L'aver lavorato al recupero del patrimonio fiabistico italiano non è stata per Calvino "un'allucinazione, una sorta di malattia professionale". Come ha dichiarato, è stata piuttosto "una conferma di qualcosa che già sapevo in partenza, [...] quell'unica convinzione mia che mi spingeva al viaggio tra le fiabe: ed è che io credo questo: le fiabe sono vere" ${ }^{[65]}$.

La pubblicazione delle Fiabe italiane è stata per Gianni Rodari, cultore della fiaba moderna, un evento storico, un trionfo della fantasia popolare italiana e l'affermazione di un momento di grande unità nazionale, perché nell'opera Italo Calvino ha raccolto il patrimonio fantastico di tutte le regioni d'Italia. Il popolo si è fatto protagonista della propria storia, il che, agli occhi di Rodari, va certamente al di sopra delle critiche sui contenuti e sui valori della fiaba, anche di quella italiana.

\section{La fiaba moderna: la lezione di Gianni Rodari}

La fiaba è stata spesso oggetto di aspre critiche, specialmente da parte di coloro che le negavano validità morale e educativa. Nel secondo dopoguerra si è verificato in Italia un vero e proprio "movimento antifiaba" che ha raggiunto il suo culmine tra gli anni Sessanta e Settanta dello scorso secolo, quando il progressivo ostracismo è arrivato al punto da eliminare le fiabe classiche dai libri di testo e dalle antologie scolastiche. Le principali obiezioni mosse alla fiaba erano essenzialmente di tipo ideologico e contestavano il suo scarso realismo, la presenza cospicua di simboli paurosi e di elementi scabrosi, la povertà di motivi, la ricorrenza di valori conservatori, ecc. Gli aspetti negativi di questo misterioso e affascinante fenomeno letterario e culturale furono evidenziati da detrattori convinti e irremovibili, che ne auspicavano la morte, ma i sostenitori entusiasti del genere fiabesco finirono infine col prevalere. Sono state soprattutto le tesi di Bettelheim a sconvolgere le posizioni negative e a incidere su un cambiamento di rotta.

[65] Calvino 1988a: 19. 
In Pro e contro la fiaba (1970), dopo aver riconosciuto nel modello fiabico la forma per eccellenza della narrativa per il bambino, Gianni Rodari ha dato una risposta sintetica e argomentata alle critiche mosse alla fiaba. La prima accusa rivolta alla fiaba è di nuocere a un'educazione moderna, perché rappresenta un'evasione dalla realtà. Rodari fa notare che la fantasia, invece, alimentata dalla fiaba, è uno strumento essenziale attraverso il quale si giunge all'essenza del reale altrettanto a fondo che mediante la scienza. La seconda accusa si ricollega alla prima e asserisce che, sostituendo l'immaginazione all'osservazione e presentando interpretazioni arbitrarie dei fenomeni, la fiaba ostacola la formazione dello spirito scientifico. Secondo Rodari, invece, proprio questa libertà di ipotesi, che sta alla base della fiaba, è uno strumento necessario all'indagine scientifica in quanto essa deve procedere spesso per supposizioni e tentativi. Alla terza accusa, relativa i particolari orridi, crudeli e sanguinosi che sono abbondantemente presenti nella fiaba e che secondo i suoi detrattori spaventerebbero inutilmente il bambino, Rodari controbatte spiegando che la paura proposta nella fiaba è uno strumento utile per far uscire allo scoperto i meccanismi di difesa. La quarta imputazione mossa alla fiaba sostiene che essa, offrendo modelli culturalmente superati, esercita nel bambino un'influenza regressiva. Rodari ammette che la concezione del mondo presentata dalla fiaba non può più servire da modello, ma ribadisce al contempo che essa è per il bambino un gioco ed in quanto tale reputa innocui certi contenuti che potrebbero avere un'influenza negativa ${ }^{[66]}$. Con la sua esaustiva difesa della fiaba, considerata il genere che aiuta il bambino a "costruirsi una mente aperta" [67], Rodari è stato colui che l'ha innovata sia come suo ri-organizzatore sia come autore che ha proposto un suo spostamento verso il fantastico. Le fiabe classiche sono presenti nella sua produzione in quanto vengono ri-usate, diventando spesso "pretesti" di nuovi testi. Rodari è pertanto un autore originale che si nutre della tradizione, soprattutto di quella connessa alla letteratura popolare, basando il suo lavoro sulla ripresa dei testi e sul loro coinvolgimento in un libero processo creativo. Sono la creatività e la fantasia a connotare l'opera

[66] Della problematica inerente la fiaba, esaminata da Rodari, si sono occupati anche Marco Dallari in La fata intenzionale. Per una pedagogia della fiaba e della controfiaba, La Nuova Italia, Firenze, 1990 e Marcello Argilli, noto inventore di fiabe moderne, che in Ci sarà una volta. Immaginario infantile e fiaba moderna, La Nuova Italia, Firenze, 1995, presenta una riflessione sulla fiaba moderna in ideale continuità con il pensiero di Gianni Rodari.

[67] Rodari 1982: 150. 
di Rodari. La tradizione vale in quanto o valorizza la creatività fantastica o serve come strumento per accenderne i processi, attraverso i percorsi della distorsione, dell'innovazione, della variazione, del rovesciamento. Ri-prendendo, ri-lanciando, ri-attivando la fiaba e dando vita a congegni narrativi fiabici moderni, traslati in un mondo meno arcaico, che si trama anzi di contemporaneità, Rodari ha espresso la libertà di rivisitare e rielaborare il patrimonio fiabico sì da renderlo più consono al nostro tempo ${ }^{[68]}$. Perché è innegabile che nel tempo sia avvenuta una sostanziale metamorfosi del bambino-ascoltatore e del bambino-lettore, dovuta all'influenza della televisione e degli audiovisivi, che hanno modificato profondamente l'immaginario infantile, in termini di arricchimento e di maggiore informazione, ma anche di condizionamenti e di omologazione. È pertanto ovvio che i nuovi processi d'informazione dell'immaginario abbiano richiesto e imposto un uso aggiornato delle fiabe popolari e la nascita al contempo di una rinnovata produzione fiabesca, maggiormente centrata sulle possibilità di trasfigurazione fantastica e più consona alla contemporaneità.

Nel marzo del 1972, su invito del comune di Reggio Emilia, Rodari tenne una serie di incontri con insegnanti di scuola dell'infanzia, elementare e media e visse un'esperienza assai significativa, sia dal punto di vista culturale che umano. Al termine degli incontri Rodari disponeva del testo di cinque conversazioni, ottenute grazie al registratore e al lavoro paziente di una dattilografa. Queste conversazioni costituiscono il materiale della Grammatica della fantasia (1973), un saggio operativo e facilmente accessibile nel quale Rodari presenta una riflessione teorica della sua attività. Ė con questo "libretto", come lo definisce l'autore, scritto "metà sul serio e metà per scherzo", che il rapporto di Rodari con i testi classici e con le loro strutture narrative si definisce meglio, e ciò avviene attraverso un più netto spostamento che mette la fantasia al centro della produzione di storie. Nelle Lezioni americane Italo Calvino aveva scritto: "La fantasia è una specie di macchina elettronica che tiene conto di tutte le combinazioni possibili e sceglie quelle che rispondono a un fine, o che semplicemente sono le più interessanti, piacevoli, divertenti" ${ }^{[69]}$. Nella

[68] Fondamentale per l'individuazione delle fonti letterarie e delle tradizioni popolari che nutrono l'opera di Rodari è il libro di Pino Boero, Una storia, tante storie. Guida all'opera di Gianni Rodari, Einaudi, Torino, 1992, la più acuta e documentata analisi dell'intera opera rodariana.

[69] Calvino 1988b: 91. 
Grammatica della fantasia Rodari teorizza questa "macchina elettronica" e cerca di scoprirne il funzionamento, mettendo a disposizione di tutti le sue considerazioni.

Quando l'opera apparve per la prima volta, a qualcuno il titolo sembrò contraddittorio e improponibile. Il titolo è difatti una sorta di ossimoro, una contraddizione, dal momento che nell'opinione comune la due parole, grammatica e fantasia, si escludono. La grammatica è, infatti, una somma di regole rigide, ferree, mentre la fantasia è sinonimo di libertà totale, di ingovernabilità, di trasgressione delle norme. In diverse occasioni Rodari ha sottolineato che tra i due termini non esiste contraddizione, e che il significato di grammatica e il significato di fantasia sono complementari e da acquisire in una prospettiva integrata. In effetti, Rodari insegna che anche la fantasia ha le sue leggi e che se davvero si vuole stimolare la creatività dei bambini, dobbiamo dare loro delle regole. Nelle prime pagine Rodari scrive: "Io spero che il libretto possa essere ugualmente utile a chi crede nella necessità che l'immaginazione abbia il suo posto nell'educazione; a chi ha fiducia nella creatività infantile; a chi sa quale valore di liberazione possa avere la parola" ${ }^{[70]}$.

Nell'opera Rodari insegna che non è più la tradizione fiabica a guidare la danza, ma la creatività, la libera fantasia o, se si vuole, la fantasia liberata. Nella Grammatica, che è una delle opere più significative di Rodari ${ }^{[71]}$, ben meditata e fondata su una profonda cultura, il fiabico viene posto in sottotono, cede il posto a tecniche surreali, che operano anche sul fiabico, decostruendolo, variandolo, distorcendolo in un'operazione di libera creatività. La fiaba diventa così strumento per produrre creatività: essa non vincola più il processo narrativo, piuttosto si fa pretesto e mezzo. Così operando, Rodari ha dato vita a un genere narrativo per l'infanzia più libero, fantastico, creativo, al centro del quale sta non più la fiaba ma, appunto, la fantasia, che è libero pensiero affabulatorio in cui il rapporto col possibile/impossibile si fa asse portante del narrare. Il pensiero creativo, nel caso di Rodari, pur nutrendosi della tradizione, si misura (e si scontra) con essa, decostruendola e spiazzandola. Per questo si può concludere che nel caso di Rodari la "tradizione non guida più il pensare narrativo,

[70] Rodari 1973: 6.

[71] È autore di racconti e filastrocche in cui ha inserito temi di riflessione morale e civile. Citiamo, tra molte, solo alcune opere: Il libro delle filastrocche (1950), Le avventure di Cipollino (1951), Filastrocche in cielo e in terra (1960), Favole al telefono (1961), C'era due volte il barone Lamberto (1978), Parole per giocare (1979). 
piuttosto vi agisce come un bacino, attraverso il quale attivare il ludus della creatività" ${ }^{[72]}$. La fiaba, sia quella popolare sia quella d'autore, si fa pretesto per il lavoro fantastico, e viene rivisitata secondo punti di vista nuovi, inediti e originali.

Il rinnovamento della fiaba tradizionale era stato avviato e messo in pratica già da Carlo Collodi, alias Carlo Lorenzini (1826-1890) con Le avventure di Pinocchio ${ }^{[73]}$, e ancora prima da Hans Christian Andersen ${ }^{[74]}$. Ma il compito di innovare la fiaba era diventato più facile nel tempo, specialmente dopo che il materiale fiabico, come osserva Rodari, era stato "catalogato, sezionato, studiato al microscopio psicologico, psicanalitico, formalistico, antropologico, strutturalistico eccetera" ${ }^{[75]}$ : per merito delle ricerche eseguite sulla fiaba da studiosi delle più disparate discipline, è stato possibile "«trattare» le fiabe classiche in una intera serie di giochi fantastici" ${ }^{[76]}$. Ė giusto ricordare che anche Rodari ha un grande debito verso uno studioso in particolare, lo psicopedagogista russo Lev Semënovič Vygotskij (1896-1934) nella cui opera, Immaginazione e creatività nell'età infantile, ha trovato interessanti spunti di riflessione sul ruolo della creatività nello sviluppo della personalità dell'individuo. Nella Grammatica confessa: "Un libretto tutto d'oro e d'argento è Immaginazione e creatività nell'età infantile di L. S. Vygotskij (Editori Riuniti, Roma 1972) che ai miei occhi, per quanto vecchiotto, ha due grandi pregi: primo, descrive con chiarezza e semplicità l'immaginazione come modo di operare della mente umana; secondo, riconosce a tutti gli uomini - e non a pochi privilegiati

[72] Catarsi 2002: 20.

[73] L'opera nacque come racconto a puntate e come tale venne pubblicata da Guido Biagi nel «Giornale per i bambini», fondato a Roma da Ferdinando Martini. La prima puntata risale al 7 luglio 1881. Sulle pagine del giornalino la storia del burattino terminava con l'impiccagione di Pinocchio al ramo della Quercia grande. Nella versione in volume, pubblicata nel 1883, per ovvi motivi di natura pedagogica, Collodi ha ampliato la storia e aggiunto altre avventure, che si concludono con il lieto fine, con la trasformazione cioè del burattino in bambino in carne e ossa. Che la pluralità di significati abbia reso l'opera un testo capace di esercitare un fascino non solo sui ragazzi, lo confermano le molteplici letture di cui è stata oggetto da studiosi di diversa matrice culturale (da E. Garroni a A. Gagliardi, da F. Cambi a R. Bertacchini, da G. Cusatelli a V. Baldacci e A. Rauch, da A. Asor Rosa a L.Volpicelli), e anche se non è questa la sede per offrire una rassegna della critica relativa, basterà prendere i volumi degli atti dei convegni collodiani di Pescia per rendersi conto della vivacità del dibattito cui il capolavoro nel tempo ha dato vita.

[74] Autore originale e singolarissimo, capace di autonome creazioni, così come di elaborare, trasfigurandoli con fervida fantasia e imprimendoli del superiore suggello dell'arte, temi e motivi della tradizione popolare, Andersen (1805-1875) traduce la propria ispirazione in un linguaggio vivo, rapido, essenziale, venato di astuzia e garbato umorismo, di grande efficacia e immediatezza rappresentativa. La raccolta più nota sono le sue Eventyr og Historier (Fiabe e racconti). Le storie di Andersen si distaccano dalle fiabe tradizionali soprattutto per la prevalenza del fantastico sulla magia. I suoi oggetti parlanti (il soldatino di piombo, la ballerina di porcellana, l'acciarino, il vecchio fanale, l'ago da rammendo, la monetina d'argento) non sono magici, ma si animano per narrare la loro storia a chi sa ascoltarla, grandi e piccoli.

[75] Rodari 1973: 52-53.

[76] Rodari 1973: 52-53. 
(gli artisti) o a pochi selezionati (a mezzo test, dietro finanziamento di qualche Foundation) - una comune attitudine alla creatività, rispetto alla quale le differenze si rivelano per lo più un prodotto di fattori sociali e culturali” ${ }^{[77]}$. Da Vygotskij Rodari ha desunto l'idea dello stretto rapporto tra attività creativa ed esperienza. Secondo lo studioso russo, l'attività creatrice dell'immaginazione deriva, infatti, dalla ricchezza dell'esperienza dell'individuo, che fornisce il materiale di cui si sostanziano le costruzioni fantastiche. Quanto più ricco è il bagaglio culturale dell'individuo, tanto più consistente è il materiale di cui può disporre la sua immaginazione: questo è il motivo per cui l'immaginazione del bambino, che non possiede ancora un ricco retroterra culturale, è più povera rispetto a quella dell'adulto. A Rodari interessa dunque capire il rapporto tra immaginazione e processo creativo al fine di individuare i procedimenti in base ai quali le competenze fantastiche funzionano e si organizzano in grammatica, in regole. Egli parte dal presupposto che la creatività si possa insegnare. Forte di questa convinzione, nella Grammatica della fantasia propone alcune tecniche molto stimolanti al fine di attivare la creatività, che in questo lavoro non sono argomento di ulteriore approfondiamo. Ci limitiamo a elencarle: $L e$ fiabe popolari come materia prima, A sbagliare storie, Cappuccetto rosso in elicottero, Le fiabe a rovescio, Insalata di favole, Fiabe a ricalco, Le carte di Propp, Fiabe in "chiave obbligata", tutte tecniche per inventare storie che muovono dal fiabico per valorizzare il fantastico e delinearlo nella sua libertà e quindi nella sua ottica decostruttiva. Il carattere divergente della scrittura di Rodari, che si manifesta nel modo in cui ha decostruito il genere fiabico con l'uso di trasgressive invenzioni verbali, nonsense, tecniche surrealistiche, giochi linguistici per attualizzarlo e adattarlo alla contemporaneità, al vissuto fantastico ed emotivo del bambino che vive e si forma in una società massmediale come la nostra, è sintetizzato in questa affermazione emblematica: "E se questa storia non ti piace com'è, / cambiala da cima a fondo, fattene un'altra da te" ${ }^{[78]}$.

La fiaba ha una chiara valenza pedagogica, è il lievito indispensabile per far crescere la fantasia e, specialmente quella moderna, riferendosi a problemi e situazioni che il bambino vive oggi, ha una più aggiornata possibilità di stimolare il pensiero divergente, che implica fluidità,

[77] Rodari 1973: 171-172.

[78] Rodari 1973: 113. 
flessibilità e originalità e riguarda essenzialmente la produzione di idee nuove. Nel discorso pronunciato in occasione del conferimento del Premio Andersen, Rodari ha detto: "Io credo che le fiabe, quelle vecchie e quelle nuove, possano contribuire ad educare la mente. La fiaba è il luogo di tutte le ipotesi: essa ci può dare le chiavi per entrare nella realtà per strade nuove, può aiutare il bambino a conoscere il mondo, gli può dare delle immagini anche per criticare il mondo" [79]. 


\section{Bibliografia}

AA.VV., Tutto è fiaba, 1980. Atti del Convegno internazionale di studi sulla fiaba, Milano, Emme Edizioni.

AA. VV., 1988. Inchiesta sulle fate. Italo Calvino e la fiaba, Bergamo, Lubrina.

Argilli Marcello, 1995. Ci sarà una volta. Immaginario infantile e fiaba moderna, Firenze, La Nuova Italia.

Bettelheim Bruno, 1997. Il mondo incantato. Uso, importanza e significati psicoanalitici delle fiabe, Milano, Feltrinelli.

Boero Pino, De Luca Carmine,1995. La letteratura per l'infanzia, Bari, Laterza.

Boero Pino, 1992. Una storia, tante storie. Guida all'opera di Gianni Rodari, Torino, Einaudi.

Boero Pino, 1999. Alla frontiera. Momenti, generi e temi della letteratura per l'infanzia, Torino, Einaudi.

Calò Anna, 1981. Il mondo della fiaba e i bisogni psicologici del bambino, Lecce, Mirella.

Calvino Italo, 1986. Fiabe italiane, Torino, Einaudi.

Calvino Italo, 1988a. Sulla fiaba, (Lavagetto Mario, a cura di), Torino, Einaudi.

Calvino Italo, 1988b. Lezioni americane, Milano, Garzanti.

Catarsi Enzo (a cura di), 2002. Gianni Rodari e la letteratura per l'infanzia, Tirrenia (Pisa), Edizioni Del Cerro.

Crispiani Piero, 1988. Andar per fiabe. Alla ricerca dell'immaginario perduto, Roma, Armando Editore.

Cusatelli Giorgio, 1983. Filologi e fiabe: le vicende grimmiane in Italia, in Studi di cultura francese ed europea in onore di Lorenza Maranini, Fasano, Schena.

Dallari Marco, 1990. La fata intenzionale. Per una pedagogia della fiaba e 
della controfiaba, Firenze, La Nuova Italia.

D’Amelio Rita, 1979. La fiaba prima finestra sul mondo, Bari, Adriatica.

Fanciulli Giuseppe, 1932. Lisa-Betta, Torino, SEI.

Ferroni Giulio, 2007. Letteratura italiana contemporanea 1945-2007, Milano, Mondadori.

Grimm Jacob e Wilhelm, 1999. Fiabe, Bergamo, Fabbri Editori.

Lüthi Max, 1982. La fiaba popolare europea. Forma e natura, Milano, Mursia.

Pisanty Valentina, 1993. Leggere la fiaba, Milano, Bompiani.

Propp Vladimir Jakovlevič, 1966. Morfologia della fiaba, Torino, Einaudi.

Propp Vladimir Jakovlevič, 2009. Le radici storiche dei racconti di magia, Roma, Newton Compton.

Rodari Gianni, 1973. Grammatica della fantasia, Torino, Einaudi.

Rodari Gianni, 1982. Il cane di Magonza, Roma, Editori Riuniti.

Salviati Carla Ida, 2002. Raccontare destini. La fiaba come materia prima di ieri e di oggi, Torino, Einaudi.

Solinas Donghi Beatrice, 1976. La fiaba come racconto, Venezia, Marsilio Edizioni. 


\section{The charm of a fairytale: the fairytale between past and present}

This paper focuses on the fairytale, usually present in all cultures, which has been entrusted, from the very beginning the task of talking to the symbolic level of the very essence of human experience. With its ancient wisdom and transparency of meaning, the fairytale meets the central questions of life: birth, love and death. The formal characteristics (absence of names, the timelessness, the abstractness of the figures and the lack of definition of space, the use of symbols, universality, etc.) allow it to transcend the historicity.

Key worlds: fairytale, fairy-tale tradition, fantasy, creativity

\section{Privlačnost bajke: bajka između prošlosti i sadašnjosti}

Ovaj je esej usredotočen na bajku, književni žanr koji je prisutan od pamtivijeka u svim kulturama. Bajka postoji u dvama oblicima: kao usmena, takozvana narodna bajka, i kao umjetnička bajka koja nastaje na temelju usvajanja, ali i preoblikovanja bitnih osobina usmene bajke. Usmene su bajke uobličene prema određenim konvencijama: odsutnost denominacija, bezvremenost, apstrakcija likova i nedostatak definicije prostora, upotreba simbola, univerzalnost. Bajka je, osobito u novije vrijeme, privukla veliku pozornost teoretičara kao složena konvencionalna književna vrsta, kako u usmenoj, tako i u pisanoj književnosti. Tako je strukturalistička analiza pripovjedne proze započela upravo analizom bajki u djelu Vladimira Proppa Morfologija bajke. Zbog moguće simbolične interpretacije, analizirali su i tumačili pojedine bajke psihoanalitički teoretičari, kao i sljedbenici Jungove analitičke psihologije.

Ključne riječi: bajka, čaroban, narodna i usmena predaja, mašta, kreativnost 
